\title{
(C) OPEN ACCESS \\ Emergency extracorporeal life support and ongoing resuscitation: a retrospective comparison for refractory out-of-hospital cardiac arrest
}

\author{
A Schober, F Sterz, H Herkner, C Wallmueller, C Weiser, P Hubner, C Testori
}

Department of Emergency Medicine, Medical University of Vienna, Waehringer Guertel, Vienna, Austria

\section{Correspondence to}

Professor F Sterz, Department of Emergency Medicine, Medical University of Vienna, Waehringer Guertel 18-20/6D - 1090

Vienna, Austria; fritz.sterz@ meduniwien.ac.at

Received 20 July 2015 Revised 30 September 2016 Accepted 10 January 2017 Published Online First 17 February 2017

\section{SLinked}

- http://dx.doi.org/10.1136/ emermed-2016-206442

\section{CrossMark}

To cite: Schober A, Sterz F, Herkner $\mathrm{H}_{\text {, et al. Emerg Med J }}$ 2017:34:277-281.

\begin{abstract}
Background In refractory cardiac arrest, with cardiopulmonary resuscitation (CPR) for more than $30 \mathrm{~min}$, chances of survival are small. Extracorporeal cardiopulmonary resuscitation (ECPR) is an option for certain patients with cardiac arrest. The aim of this study was to evaluate characteristics of patients selected for ECPR. Methods Anonymised data of adult patients suffering refractory cardiac arrest, transported with ongoing CPR to an ED of a tertiary care centre between 2002 and 2012 were analysed. Outcome measure was the selection for ECPR. Secondary outcome was 180 days survival in good neurological condition.

Results Overall, 239 patients fulfilled the inclusion criteria. ECPR was initiated in seven patients. Patients treated with ECPR were younger (46 vs 60 years; $p=0.04$ ), had shorter intervals before CPR was started ( 0 vs $1 \mathrm{~min} ; \mathrm{p}=0.013$ ), faster admissions at the ED (38 vs $56 \mathrm{~min} ; p=0.31$ ) and lower blood glucose levels on admission ( $14 \mathrm{vs} 21 \mathrm{mmol} / \mathrm{L} ; \mathrm{p}=0.018$ ). Survival to discharge in good neurological condition was achieved in $14(6 \%)$ of all patients. One patient in the ECPR group survived in excellent neurological condition. Age was independently associated with the selection for ECPR (OR 0.07; 95\% Cl 0.01 to $0.85 ; p=0.037$ ).

Conclusions Emergency extracorporeal life support was used for a highly selected group of patients in refractory cardiac arrest. Several parameters were associated with the decision, but only age was independently associated with the selection for ECPR. The patient selection resulting in a survival of one patient out of seven treated seems reasonable. Randomised controlled trials evaluating the age limit as selection criteria are urgently needed to confirm these findings.
\end{abstract}

\section{INTRODUCTION}

Outcome after out-of-hospital cardiac arrest (OOHCA) is poor and despite measures to advance the treatment quality, survival rate and neurological recovery need further improvement. Currently, overall survival ranges around $10 \% .^{1-3}$ The guidelines for resuscitation focus on the early recognition of cardiac arrest, fast deployment of sufficient basic life support as well as defibrillation and standardised postresuscitation care including therapeutic hypothermia to assure the best possible outcome. ${ }^{4}$ With the goal of further improving outcome after cardiac arrest, several attempts have been made to strengthen the links in the chain of survival and new ALS techniques and treatment approaches as well as specialised centres for post-cardiac arrest care are the subject of research and discussion. ${ }^{3}$

\section{Key messages}

What is already known on this subject? Current literature suggests a benefit for patients in refractory cardiac arrest treated with extracorporeal cardiopulmonary resuscitation (ECPR). Evidence concerning selection criteria for this intervention and outcome in real life use is still scarce.

What might this study add?

In this retrospective analysis of 239 patients in refractory cardiac arrest, seven were treated with ECPR and one survived with good neurological outcome. Younger age was independently associated with being selected for ECPR. ECPR might be a valuable treatment option for highly selected patients.

Extracorporeal cardiopulmonary resuscitation (ECPR) using extracorporeal life support is one of the most controversial treatment options in OOHCA due to its ability to treat a potentially reversible underlying condition. It is a costly and resource-intensive procedure, and there are no randomised trials. Several studies have been published which show positive results in patients resuscitated with ECPR.$^{8-14}$ Outcome shows significant variation between those studies with survival in favourable neurological condition ranging from $4 \%$ to $54 \%$. Clearly, patient selection seems to be a key issue together with the speed of the performance to safe precious time.

The aim of this study was to provide data regarding characteristics of refractory cardiac arrest patients selected for ECPR at our ED.

\section{METHODS}

This study is a retrospective cohort analysis based on a prospectively designed and conducted registry of patients resuscitated from cardiac arrests in an urban population and admitted to an ED of a tertiary care centre. The institutional Ethical Committee of the Medical University of Vienna has approved this registry (1814/2012), and the procedures were in accordance with the ethical standards.

Adult patients suffering refractory OOHCA due to cardiac origin were eligible; refractory arrest was defined as $30 \mathrm{~min}$ of ongoing resuscitation, without occurrence of return of spontaneous circulation (ROSC according to Utstein criteria) prior 
to admission. ${ }^{15} 16$ Patients who had a clear clinical indication for the use of ECPR (ie, severe hypothermia) were excluded. The eligible period was February 2002 until January 2012 to allow for a homogenous patient population, since therapeutic hypothermia as a part of standardised postresuscitation care was established as of 2002 at our department. A flow diagram of patient inclusion is shown in figure 1.

CPR was performed according to the international recommendations available at that time. ${ }^{17-19}$ Quality feedback for CPR measures was provided via structured feedback mechanism reported elsewhere. ${ }^{19}$ Mechanical CPR was routinely used (Autopulse System (Zoll Medical, Massachusetts, USA) and Lund University Cardiopulmonary Assist System (LUCAS2, Physio Control, Washington, USA)) to facilitate transport CPR and continued at the ED during ECPR implantation. After femoro-femoral cannulation, venoarterial extracorporeal life support via extracorporeal membrane was performed at the ED (ECPR). The device consisted of a centrifugal pump (Bio-Pump Plus, Medtronic, USA or Cardiohelp, Maquet, Germany). Cannulation was either performed percutaneously, using Seldinger technique, or surgically, with a cutdown to the femoral vessels. In either method of cannulation. it was preferred to establish venous and arterial access on the ipsilateral side. Cannulae size was determined by estimation in percutaneous access and according to vessel size in surgical access. Setting and management, such as out of hospital and postresuscitation care, the finding of the decision for ECPR, the cannulas and bypass pumps, oxygenators plus heat exchanger used and set up have been described by Wallmüller et $a l^{20}$ There was no structured protocol for ECPR and the decision was at the discretion of the treating emergency physician.

In every cardiac arrest case in our department, trained study nurses and physicians use a special case report form to prospectively collect data for a prospective cardiac arrest database. All data are documented prospectively at the time of the arrest according to 'Utstein Style Criteria' ${ }^{21}$ with structured protocols on demographic factors, chronic pre-arrest health conditions and resuscitation details. In witnessed cardiac arrest with bystander CPR or cardiac arrest witnessed by medical professionals and immediate ALS, a 'no-flow interval' was calculated (time of cardiac arrest to the time of chest compressions). Similarly, a 'low-flow interval' was calculated from witnessed cardiac arrest to either ROSC or established ECPR. Data were entered into the cardiac arrest registry database with range and consistency checks. This database was used for the analysis.

Study nurses and physicians obtained 6-month mortality rates and neurological assessment via telephone calls, contacting the patients or their legal deputies for follow-up information. Neurological assessment was accomplished using cerebral performance categories (CPC), which were classified as follows:

(1) good cerebral performance, (2) moderate cerebral disability, (3) severe cerebral disability, (4) coma and (5) brain death. Good outcome was defined as 180 days survival and CPC 1 or 2 , at 6 months after cardiac arrest.

Continuous data are presented as median and 25\% to $75 \%$ IQR. Categorical data are presented as count and relative frequency. Patients were stratified according to outcome (good vs bad outcome) and the exposure to ECPR versus conventional
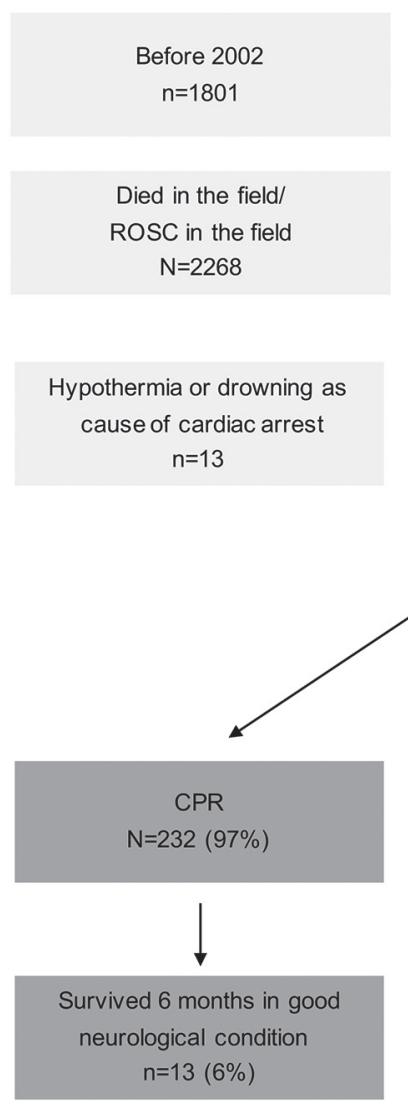
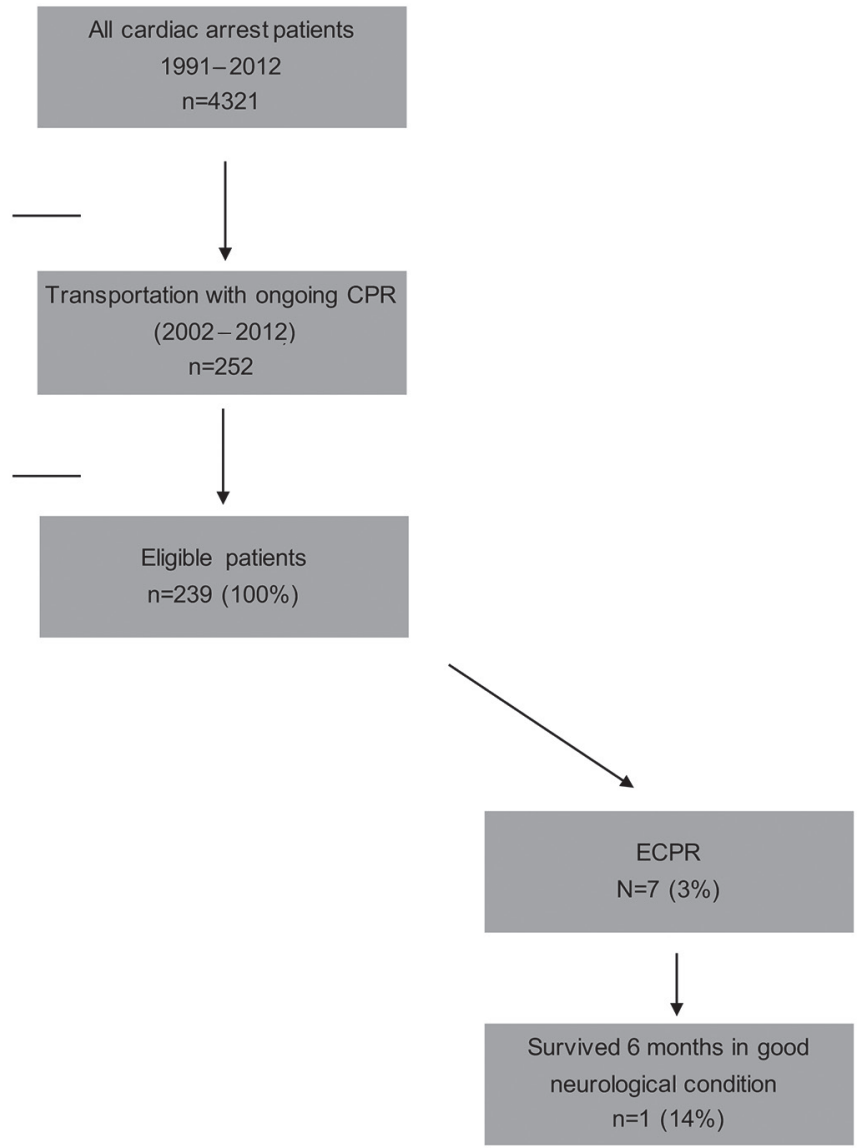

Figure 1 Flow chart of patient inclusion. CPR, cardiopulmonary resuscitation; ECPR, extracorporeal cardiopulmonary resuscitation; ROSC, return of spontaneous circulation. 
CPR. These groups were compared with regard to baseline characteristics and resuscitation-related factors with univariate analyses, using Student's t test and Mann-Whitney $U$ test for continuous factors and Fisher's exact test for categorial variables as appropriate. To determine the association of variables with exposure to the treatment 'ECPR', we used multivariable logistic regression models to calculate ORs with 95\% CIs. For hypothesis testing, we used the Wald test. Candidate variables were chosen a priori or if the $\mathrm{p}$ value in the univariate analyses was $>0.20$. For multivariable modelling, we categorised continuous variables and handled missing values as a separate category. We tested for first-order interactions. For data management and analyses, we used MS Excel for Mac (Microsoft, Redmond, California, USA), SPSS Statistics 20 (IBM, Armonk, New York, USA) and Stata 11 for Mac (Stata, College Station, Texas, USA). Generally, a two-sided p value $<0.05$ was considered statistically significant.

\section{RESULTS}

Among 4321 cardiac arrest registry files during the study period, 252 patients were admitted to our department in refractory cardiac arrest. Of those, 13 patients had other causes of cardiac arrest (hypothermia and drowning). Thus, a cohort of 239 patients remained for further analysis (figure 1). Of those, $232(97 \%)$ patients received conventional CPR and were treated according to the current guidelines of adult CPR and eventually postresuscitation care, if they achieved ROSC. ${ }^{16}$ In 7 patients (3\%), emergency extracorporeal life support was established after admittance.

The baseline characteristics of all patients as well as the characteristics for the cohort treated with standard CPR compared with those treated by ECPR are shown in table 1.

Conventional CPR was performed over a median of 86 (IQR 79-116) $\mathrm{min}$ before patients could be supported by ECPR. Time from admission to ECPR was 55 (IQR 48-65) min. Vascular access strategy consisted of surgical implantation in three and cannulation using 'Seldinger' technique in four patients. The median time needed for cannulation was 21 (15-63) min. Runtime of ECPR after initiation was a median of 15 (4-83) hours. ECPR-related adverse events were observed in two patients. In one patient, both ECPR cannulae were placed in the same femoral artery and ECPR subsequently failed, and in the second patient surgical revision of the cannulation site had to be performed.

Median time from cardiac arrest to ED admission was $56 \mathrm{~min}$ (39-72) and was significantly shorter for patients suffering from cardiac arrest in a public place (46 vs $73 \mathrm{~min}, \mathrm{p}<0.001)$. It seemed to be shorter for patients receiving ECPR (38 vs $56 \mathrm{~min}$ ), but the results did not reach significance.

Overall, 73 patients (31\%) achieved ROSC: 3/7 (43\%) with ECPR and 70/232 (30\%) with conventional CPR, with median time from admission to ROSC being 17 (IQR 8-27) $\mathrm{min}$ ). Survival for 6 months in good neurological condition was achieved in $14(6 \%)$ patients with 1 patient from the 7 in the ECPR groups (14\%) and 13/232 (6\%) in the CPR group. The results did not reach statistical significance (OR 2.8; 95\% CI 0.3 to $25, \mathrm{p}=0.34)$. Generally, survivors had shorter time to admission (32 vs $58 \mathrm{~min}$; $\mathrm{p}=0.01$ ), initial serum lactate was lower (11 vs $15 \mathrm{mmol} / \mathrm{L} ; \mathrm{p}=0.01$ ) and serum potassium on admission was lower (3.9 vs $4.7 \mathrm{mmol} / \mathrm{L} ; \mathrm{p}=0.04)$ in univariate analysis. The sole survivor in the ECPR cohort was a male patient (59 years), who suffered witnessed CA in a public place. He received conventional CPR during transport for $32 \mathrm{~min}$. The percutaneous cannulation took $19 \mathrm{~min}$ after admission to our ED. After 3 days of intensive care, he woke up and could be transferred to

\begin{tabular}{|c|c|c|c|c|}
\hline & $\begin{array}{l}\text { Total } \\
\mathrm{n}=239 \\
\end{array}$ & $\begin{array}{l}\text { ECPR } \\
\mathrm{n}=7\end{array}$ & $\begin{array}{l}\text { Conventional CPR } \\
\mathrm{n}=232\end{array}$ & $\mathrm{p}$ Value \\
\hline Female & $61(26)$ & $2(28)$ & $59(25)$ & 0.571 \\
\hline Age (years) & $60(50-69)$ & $46(31-59)$ & $60(50-70)$ & 0.044 \\
\hline CHF & $30(13)$ & 0 & $30(13)$ & 0.601 \\
\hline Smoker & $58(24)$ & $1(14)$ & $57(25)$ & 0.461 \\
\hline CAD & $66(28)$ & $1(14)$ & $65(28)$ & 0.677 \\
\hline COPD & $24(10)$ & $1(14)$ & $23(10)$ & 0.528 \\
\hline Diabetes mellitus & $44(18)$ & 0 & $44(19)$ & 0.355 \\
\hline Arterial hypertension & $70(29)$ & $2(28)$ & $68(29)$ & 0.99 \\
\hline CPC 1 or 2 before CA & $235(99)$ & $7(100)$ & $228(99)$ & 0.99 \\
\hline Witnessed CA & $210(88)$ & $6(86)$ & $204(88)$ & 0.601 \\
\hline CA at home & $92(39)$ & $1(14)$ & $91(39)$ & 0.255 \\
\hline CA during transportation & $41(18)$ & $2(29)$ & $39(17)$ & 0.344 \\
\hline VF/VT, initially recorded & $138(58)$ & $4(57)$ & $134(58)$ & 0.631 \\
\hline $\begin{array}{l}\text { Bystander CPR } \\
\text { performed }\end{array}$ & $73(31)$ & $2(28)$ & $71(31)$ & 0.99 \\
\hline Defibrillations during ALS & $4(1-10)$ & $3(0-6)$ & $4(1-10)$ & 0.552 \\
\hline $\begin{array}{l}\text { Epinephrine applied } \\
\text { during CPR (mg) }\end{array}$ & $9(6-12)$ & $9(6-14)$ & $8(6-12)$ & 0.518 \\
\hline $\begin{array}{l}\text { 'No-flow' time interval } \\
\text { (minutes) if arrest was } \\
\text { witnessed }\end{array}$ & $1(0-6)$ & $0(0-1.5)$ & $1(0-6)$ & 0.013 \\
\hline $\begin{array}{l}\text { Time from CA to } \\
\text { admission (minutes) }\end{array}$ & $56(39-72)$ & $38(27-66)$ & $56(40-72)$ & 0.31 \\
\hline $\begin{array}{l}\text { Total CPR time (min) if } \\
\text { arrest was witnessed }\end{array}$ & 78 (59-97) & 97 (79-147) & 77 (58-95) & 0.093 \\
\hline $\begin{array}{l}\text { Time from admission } \\
\text { to ECPR/ROSC (min) }\end{array}$ & & $55(45-68)$ & $17(8-27)$ & \\
\hline Any ROSC & $93(39)$ & $4(57)$ & $89(38)$ & 0.436 \\
\hline Serum $\mathrm{pH}$ on admission & $\begin{array}{l}6.92(6.80- \\
7.02)\end{array}$ & $\begin{array}{l}6.95(6.91- \\
7.10)\end{array}$ & $6.92(6.80-7.02)$ & 0.15 \\
\hline $\begin{array}{l}\text { Serum lactate on } \\
\text { admission (mmol/L) }\end{array}$ & $\begin{array}{l}14.6 \\
(11-17)\end{array}$ & $12(9-21)$ & $15(11-17)$ & 0.122 \\
\hline $\begin{array}{l}\text { Serum glucose levels on } \\
\text { admission (mmol/L) }\end{array}$ & $21(15-26)$ & $14(7-18)$ & $21(15-26)$ & 0.018 \\
\hline $\begin{array}{l}\mathrm{paCO}_{2} \text { levels on } \\
\text { admission }(\mathrm{mm} \mathrm{Hg})\end{array}$ & $62(43-77)$ & $38(18-63)$ & $62(43-77)$ & 0.37 \\
\hline $\begin{array}{l}\mathrm{paO}_{2} \text { levels on admission } \\
(\mathrm{mm} \mathrm{Hg})\end{array}$ & $\begin{array}{l}85(59- \\
147)\end{array}$ & $82(28-158)$ & $85(61-147)$ & 0.33 \\
\hline $\begin{array}{l}\text { Core temperature } \\
\text { admission }\left({ }^{\circ} \mathrm{C}\right)\end{array}$ & $35(34-36)$ & $35(34-36)$ & $35(34-36)$ & 0.313 \\
\hline $\begin{array}{l}\text { Therapeutic hypothermia } \\
\text { initiated }\end{array}$ & $51(21)$ & $3(43)$ & $48(21)$ & $>0.5$ \\
\hline $\begin{array}{l}\text { Coronary intervention } \\
\text { performed }\end{array}$ & $13(5)$ & $2(28)$ & $11(5)$ & 0.049 \\
\hline
\end{tabular}

Data presented as $\mathrm{n}$ and (\%) or median and (IQR) as appropriate.

$C A$, cardiac arrest; $C A D$, coronary artery disease; $C H F$, chronic heart failure; $C O P D$ chronic obstructive pulmonary disease; CPC, cerebral performance category $1-5$; CPR, cardiopulmonary resuscitation;ECPR, extracorporeal cardiopulmonary resuscitation; VF, ventricular fibrillation; $\mathrm{VT}$, ventricular tachycardia.

a normal ward after 1 week. Finally, he could be discharged in excellent neurological condition after 20 days, showing CPC 1 at 6 months follow-up.

Patients in the ECPR cohort were younger, the time without basic life support ('no-flow' time) was shorter, blood glucose levels on admission were lower and coronary intervention and therapeutic hypothermia was performed more often as displayed in table 1 . The first recorded heart rhythm did not differ between the groups. Neither did the rate of witnessed cardiac arrest or bystander CPR. The only factor significantly associated with 
Table 2 Factors associated with the exposure to ECPR in multivariable logistic regression analysis

\begin{tabular}{llll}
\hline & OR & $95 \%$ Cl & p Value \\
\hline Age (<60 vs 60+ years) & 0.07 & 0.01 to 0.85 & 0.037 \\
Female gender (yes vs no) & 2.63 & 0.27 to 26.15 & 0.41 \\
\hline No-flow time (<1 vs 1+ min) & 0.07 & 0.01 to 1.22 & 0.068 \\
\hline Shockable heart rhythm (yes vs no) & 3.2 & 0.28 to 35.65 & 0.35 \\
Witnessed CA (yes vs no) & 0.01 & 0 to 2.47 & 0.10 \\
\hline Basic life support performed (yes vs no) & 1.36 & 0.14 to 13.22 & 0.79 \\
\hline pH on admission (<6.92 vs 6.92+) & 0.73 & 0.31 to 1.70 & 0.47 \\
\hline Lactate on admission (<14.6 vs 14.6+ mmol/L) & 1.01 & 0.82 to 1.27 & 0.86 \\
\hline Public place CA (yes vs no) & 0.73 & 0.06 to 9.1 & 0.80 \\
\hline
\end{tabular}

CA, cardiac arrest; ECPR, extracorporeal cardiopulmonary resuscitation; no-flow time, interval from cardiac arrest to CPR dichotomised at the median.

the use of ECPR in multivariate logistic regression analysis was age $(\mathrm{OR}=0.07 ; 95 \% \mathrm{CI} 0.01$ to $0.85 ; \mathrm{p}=0.037)$. All factors are displayed in table 2.

\section{DISCUSSION}

This report presents our experience with patients who were admitted under ongoing CPR without sustained return of spontaneous circulation and were either treated by conventional ALS or ECPR. Since patient selection and a rapid procedural performance are key issues, we analysed with regard to selection for ECPR and we found patients treated by ECPR to be younger, to have shorter no-flow time intervals and less metabolic derangement on admission, in univariate analyses. Surprisingly, the initial heart rhythm as well as the rate of bystander CPR, the lactate levels and the $\mathrm{pH}$ on admission did not differ between the groups, and were therefore probably not used for patient selection. ECPR-treated patients underwent coronary angiography and therapeutic hypothermia more often, which might rather reflect a chain of events than a cause-and-effect relationship. Because ECPR is a resource intensive procedure, patient-related factors and organisational concerns and capabilities at time of admission might have influenced the decision concerning ECPR.

One out of seven patients treated with ECPR survived, suggesting the potential for better survival in the ECPR group, but this did not reach significance in univariate testing and needs to be interpreted with care due to the small sample size, and the variety of potential confounders. The surviving patient in the ECPR group also had optimal time management.

The principal limitation of this study may be its retrospective nature. However, resuscitation-related factors were closely monitored in both groups during this specific time interval, reducing the probability of a bias. The absence of a strict protocol may furthermore lead to potential confounding with regard to the outcome of the selected patients, but the goal of this analysis was to identify factors used for patient selection. The fairly small sample size is another limitation in this study but we focused on this population specifically to analyse a homogeneous cohort of patients with ECPR with a cardiopulmonary cause of cardiac arrest and ongoing resuscitation at admission. The low number of patients receiving ECPR also limits our ability to do more in-depth statistical analyses. In addition to the patient and resuscitation-related parameters, other factors might as well have influenced the selection, such as unavailability of the ECPR system or a lack of resources. The outcome of all patients with ECPR treated at our ED was reported previously by Wallmüller et $a l^{20}$
Comparing the findings of our study with the current literature, we generally found similar criteria used for ECPR selection in our cohort, especially the factors being significant in univariate analyses. The group of Nagao in Japan ${ }^{12}$ analysed outcomes of 50 patients who underwent a treatment bundle of ECPR, therapeutic hypothermia and coronary intervention. Patients had to have witnessed cardiac arrest and were selected by age (18--74 years), and initial ECG (shockable rhythm). Apart from the shockable heart rhythm, which is generally associated with better outcome, but did not reach any significance in our analyses, these results are in accordance with our findings. Chen et al published two, fairly large trials, comparing conventional CPR with ECPR in a retrospective, propensity score-matched analysis. ${ }^{89}$ The selection criteria in these studies were in accordance with our findings. The age cut-off was at 75 years, with a median age of 57 years for ECPR patients. Furthermore, they did not restrict ECPR to shockable heart rhythms with 51\% of patients with ECPR presenting in a non-shockable initial rhythm. The cardiac arrest to ECPR time interval in these studies was impressively short, and the definition of refractory cardiac arrest at $10 \mathrm{~min}$ does not reflect our current algorithms. Fagnoul et al recently published an overview of ECPR use in in and out-of-hospital cardiac arrest. They found the survival rates for ECPR in patients with OOHCA to be 15\%-20\% provided that time to ECPR did not exceed the 'golden hour' ${ }^{10}$ Our experience seems to support this conclusion, considering the patient achieving good outcome in the ECPR cohort had an interval from cardiac arrest to ECPR of $51 \mathrm{~min}$, respectively. A first prospective trial was published last year by Stub et al, reporting a total of 26 patients treated with ECPR (11 of them after refractory OOHCA). ${ }^{14}$ The authors found overall survival in neurologically intact condition in 14/26 (54\%). These positive results may be due to a strict patient selection (age 52 $(38 ; 60)$; ventricular fibrillation in $72 \%)$ and excellent procedure and time management, taking the short interval from arrest to ECPR $(56 \mathrm{~min}(40 ; 85))$ into consideration. We found a lower survival rate in our cohort, even though the patients seem to be comparable in age. This might be caused by the significantly longer delay from arrest to ECPR in our patients. Lamhaut et al reported promising results of the first seven cases of refractory OOHCA treated by out-of-hospital deployed ECPR. ${ }^{22}$ They were able to start ECPR outside the hospital within the 'golden hour'. Our data show a median time to admittance of more than $70 \mathrm{~min}$ in patients who suffered cardiac arrest in a private home. Therefore, measures to decrease that delay as much as possible are needed when admitting patients in refractory arrest for ECPR in our setting. Out-of-hospital ECPR could save precious time in cases of difficult patient rescue and transport, since 1 hour from cardiac arrest has been established as the cut-off for this intervention.

\section{CONCLUSION}

Concluding from our findings, the decision to use ECPR seems to be influenced by resuscitation and patient-related factors, such as the age of the patient, the time of circulatory standstill without basic life support and metabolic derangement on arrival. One of seven ECPR-treated patients survived in excellent condition, which makes the use of these parameters reasonable to guide future decisions concerning patient selection for this highly sophisticated intervention. Randomised, controlled clinical trials, as currently being conducted in Prague ${ }^{23}$ and Vienna (clincialtrials.gov NCT01605409), are nevertheless urgently needed to gain evidence whether ECPR should become a standard treatment in these carefully selected patients. 


\section{Key points}

- Extracorporeal cardiopulmonary resuscitation is a sophisticated treatment option providing good outcome in a highly selected patient population with cardiac arrest.

- It might be reasonable to restrict its use to situations, which allow short transport intervals to the hospital to further improve the outcome.

Acknowledgements We want to thank the team of the Department of Emergency Medicine, Medical University of Vienna, for their efforts and contributions.

Contributors The study was planned and designed by AS, CWA, FS, CT and HH. $\mathrm{AS}, \mathrm{CT}, \mathrm{CWA}, \mathrm{CWE}$ and $\mathrm{PH}$ carried out the data acquisition. $\mathrm{HH}$ performed statistical analysis with critical revision and substantial contributions provided by AS and FS. Interpretation of data was mainly carried out by AS, CT, and FS. AS, and CT drafted the manuscript with substantial contribution of CWA, CWE, FS, PH and $\mathrm{HH}$. All authors read, provided crtitical revision to and approved the final manuscript. All authors agree to be accountable for all aspects of the work in ensuring that questions related to the accuracy or integrity of any part of the work are appropriately investigated and resolved. Each author has participated sufficiently in the work to take public responsibility for appropriate portions of the content.

Competing interests None declared.

Provenance and peer review Not commissioned; externally peer reviewed.

Open Access This is an Open Access article distributed in accordance with the Creative Commons Attribution Non Commercial (CC BY-NC 4.0) license, which permits others to distribute, remix, adapt, build upon this work non-commercially, and license their derivative works on different terms, provided the original work is properly cited and the use is non-commercial. See: http://creativecommons.org/ licenses/by-nc/4.0/

(c) Article author(s) (or their employer(s) unless otherwise stated in the text of the article) 2017. All rights reserved. No commercial use is permitted unless otherwise expressly granted.

\section{REFERENCES}

1 Herlitz J, Bahr J, Fischer M, et al. Resuscitation in Europe: a tale of five European regions. Resuscitation 1999:41:121-31.

2 Herlitz J, Ekström L, Wennerblom B, et al. Effect of bystander initiated cardiopulmonary resuscitation on ventricular fibrillation and survival after witnessed cardiac arrest outside hospital. Br Heart J 1994;72:408-12.

3 Sunde $K$, Pytte M, Jacobsen D, et al. Implementation of a standardised treatment protocol for post resuscitation care after out-of-hospital cardiac arrest. Resuscitation 2007;73:29-39.

4 Nolan JP, Soar J, Zideman DA, et al. European resuscitation council guidelines for resuscitation 2010 section 1. executive summary. Resuscitation 2010;81:1219-76.

5 Nolan JP, Soar J. Post resuscitation care--time for a care bundle? Resuscitation 2008;76:161-2.

6 Donnino MW, Rittenberger JC, Gaieski D, et al. The development and implementation of cardiac arrest centers. Resuscitation 2011;82:974-8.

7 Lurie KG, Idris A, Holcomb JB. Level 1 cardiac arrest centers: learning from the trauma surgeons. Acad Emerg Med 2005;12:79-80.
8 Chen YS, Lin JW, Yu HY, et al. Cardiopulmonary resuscitation with assisted extracorporeal life-support versus conventional cardiopulmonary resuscitation in adults with in-hospital cardiac arrest: an observational study and propensity analysis. Lancet 2008:372:554-61.

9 Chen YS, Yu HY, Huang SC, et al. Extracorporeal membrane oxygenation support can extend the duration of cardiopulmonary resuscitation. Crit Care Med 2008:36:2529-35.

10 Fagnoul D, Combes A, De Backer D. Extracorporeal cardiopulmonary resuscitation. Curr Opin Crit Care 2014;20:259-65.

11 Sakamoto T, Morimura N, Nagao K, et al; SAVE-J Study Group. Extracorporeal cardiopulmonary resuscitation versus conventional cardiopulmonary resuscitation in adults with out-of-hospital cardiac arrest: a prospective observational study. Resuscitation 2014;85:762-8.

12 Nagao K, Hayashi N, Kanmatsuse K, et al. Cardiopulmonary cerebral resuscitation using emergency cardiopulmonary bypass, coronary reperfusion therapy and mild hypothermia in patients with cardiac arrest outside the hospital. J Am Coll Cardiol 2000;36:776-83.

13 Morimura N, Sakamoto T, Nagao K, et al. Extracorporeal cardiopulmonary resuscitation for out-of-hospital cardiac arrest: A review of the japanese literature. Resuscitation 2011;82:10-14.

14 Stub D, Bernard S, Pellegrino V, et al. Refractory cardiac arrest treated with mechanical CPR, hypothermia, ECMO and early reperfusion (the CHEER trial). Resuscitation 2015;86:88-94.

15 Cummins RO, Chamberlain DA, Abramson NS, et al. Recommended guidelines for uniform reporting of data from out-of-hospital cardiac arrest: the Utstein style. A statement for health professionals from a task force of the American Heart Association, the European Resuscitation Council, the Heart and Stroke Foundation of Canada, and the Australian Resuscitation Council. Circulation 1991:84:960-75.

16 Nolan JP, Soar J, Zideman DA, et al; ERC Guidelines Writing Group. European resuscitation council guidelines for resuscitation 2010 section 1. executive summary. Resuscitation 2010;81:1219-76.

17 Nolan JP, Deakin CD, Soar J, et al; European Resuscitation Council. European resuscitation council guidelines for resuscitation 2005. section 4. adult advanced life support. Resuscitation 2005;67(Suppl 1):S39-86.

18 Anon. Part 6: advanced cardiovascular life support. Section 8: postresuscitation care. European Resuscitation Council. Resuscitation 2000;46:195-201.

19 Nürnberger A, Sterz F, Malzer R, et al. Out of hospital cardiac arrest in Vienna: incidence and outcome. Resuscitation 2013;84:42-7.

20 Wallmüller C, Sterz F, Testori C, et al. Emergency cardio-pulmonary bypass in cardiac arrest: seventeen years of experience. Resuscitation 2013;84:326-30.

21 Jacobs I, Nadkarni V, Bahr J, et al; International Liason Committee on Resusitation. Cardiac arrest and cardiopulmonary resuscitation outcome reports: update and simplification of the utstein templates for resuscitation registries. A statement for healthcare professionals from a task force of the international liaison committee on resuscitation (American Heart Association, European Resuscitation Council, Australian Resuscitation Council, New Zealand Resuscitation Council, Heart and Stroke Foundation of Canada, InterAmerican Heart Foundation, Resuscitation Council of Southern Africa). Resuscitation 2004;63:233-49.

22 Lamhaut L, Jouffroy R, Soldan M, et al. Safety and feasibility of prehospital extra corporeal life support implementation by non-surgeons for out-of-hospital refractory cardiac arrest. Resuscitation 2013;84:1525-9.

23 Belohlavek J, Kucera K, Jarkovsky J, et al. Hyperinvasive approach to out-of hospital cardiac arrest using mechanical chest compression device, prehospital intraarrest cooling, extracorporeal life support and early invasive assessment compared to standard of care. A randomized parallel groups comparative study proposal. "Prague OHCA study". J Trans/ Med 2012;10:163. 